\title{
Asymptotic Theory for Quantum Bose Systems with Many Degrees of Freedom
}

\author{
Misha Vishik \\ Department of Mathematics \\ The University of Texas at Austin \\ Austin, TX 78712-1082, U.S.A. \\ Gennady Berman \\ Los Alamos National Laboratory, MS B213 \\ Los Alamos, NM 87545, U.S.A. I \\ (Dated: January 16, 2019)
}

\begin{abstract}
We construct asymptotic expansions of Laplace type for the time-dependent quantum averages for Bose systems with many degrees of freedom, initially populated in coherent states. These solutions are localized in phase space, and they are different from the usual oscillating asymptotics for the quasi-classical wave functions. These expansions are valid on any fixed time interval, and caustics do not contribute to the asymptotics.

PACS numbers: 03.65.-w; 42.50.-p; 74.20.-z
\end{abstract}

In the present paper we develop an asymptotic theory for the partial differential equations describing the evolution of averages in coherent states as $\hbar \rightarrow 0$, for Bose sistems with many degrees of freedom. The crucial observation is that Laplace asymptotics, describing the quantities exponentially localized in the parameter of a coherent state and not the standard oscillating WKB asymptotics play the central role in the theory. Remarkably the phase and the amplitude in Laplace asymptotic expansion can be constructed using the classical Hamilton-Jacobi theory without a usual difficulty associated with caustics. In a sense the expansion we describe below corresponds to a "purely imaginary" WKB with a nonnegative phase. We use coherent states as initial wave functions of the evolution. Coherent states are the closest states to the classical systems because they realize the minimum uncertainty relation. They were introduced in R.J. Glauber [1]. Different approaches to the dynamical quasi-classical theory were reviewed in [2], [3].

Our theory is based on the algebra of non-commutative operators which was discussed in detail in $\sqrt[4]{4}$, , 迎, 6], [7], [8]. Mathematical foundations of the theory are thoroughly developed in the works of V.P. Maslov [9], [10], L. Hörmander 111 and many other authors. The theory we develop below is formulated in a general form and can be applied to rather general Bose systems with many degrees of freedom. In particular, it can be used for computing dynamical quantum effects in complicated Bose-Einstein condensate systems.

\section{1.}

We consider a time-independent Hamiltonian

$$
H\left(a_{1}^{\dagger}, \ldots, a_{N}^{\dagger}, a_{1}, \ldots, a_{N}\right)=\sum_{\ell, s} H_{\ell s} a^{\dagger \ell} a^{s}
$$

where

$$
\ell=\left(\ell_{1}, \ldots, \ell_{N}\right) \in \mathbb{Z}_{+}^{N}, \quad s=\left(s_{1}, \ldots, s_{N}\right) \in \mathbb{Z}_{+}^{N}
$$

$\mathbb{Z}_{+}$stands for the set of nonnegative integers; $a^{\dagger \ell}=a_{1}^{\dagger \ell_{1}} \cdots a_{N}^{\dagger \ell_{N}} ; a^{s}=a_{1}^{s_{1}} \cdots a_{N}^{s_{N}}$. Here as usual

$$
a_{k}^{\dagger}=\frac{1}{\sqrt{2}}\left(x_{k}-\hbar \frac{\partial}{\partial x_{k}}\right), \quad a_{k}=\frac{1}{\sqrt{2}}\left(x_{k}+\hbar \frac{\partial}{\partial x_{k}}\right)
$$

are the creation operator and the annihilation operator respectively; $\left[a_{k}^{\dagger}, a_{\ell}\right]=-\delta_{k \ell} \hbar$ for $k, \ell=1, \ldots, N$.

We assume $H$ is formally self-adjoint, i.e.,

$$
H_{\ell s}=H_{s \ell}^{*}
$$


The Heisenberg equation for an arbitrary operator valued function $F(t)$ is

$$
\dot{F}=\frac{i}{\hbar}[H, F]
$$

We introduce Poisson vectors $\Phi_{\alpha}$ and coherent states $|\alpha\rangle$ as follows: for any $\alpha=\left(\alpha_{1}, \ldots, \alpha_{N}\right) \in \mathbb{C}^{N}$

$$
\begin{aligned}
& \Phi_{\alpha}=(\pi \hbar)^{-N / 4} \exp \left\{-\frac{1}{2 \hbar}\left(x^{2}-2 \sqrt{2} x \cdot \alpha+\alpha^{2}\right)\right\} \\
& |\alpha\rangle=\exp \left(-\frac{|\alpha|^{2}}{2 \hbar}\right) \Phi_{\alpha} .
\end{aligned}
$$

Then, $a \Phi_{\alpha}=\alpha \Phi_{\alpha}$, i.e.,

$$
\begin{aligned}
a_{k} \Phi_{\alpha} & =\alpha_{k} \Phi_{\alpha}, & k & =1, \ldots, N . \text { Also } \\
\hbar \frac{\partial}{\partial \alpha_{k}} \Phi_{\alpha} & =a_{k}^{\dagger} \Phi_{\alpha}, & k & =1, \ldots, N .
\end{aligned}
$$

Unlike $\Phi_{\alpha}$, the vectors $|\alpha\rangle$ are normalized: $\langle\alpha \mid \alpha\rangle=1$. Notice that $\Phi_{\alpha}$ is holomorphic with respect to $\alpha \in \mathbb{C}^{N}$. Our goal is to study the expectation value $\langle\alpha|F(t)| \alpha\rangle$. To obtain the partial differential equation for this quantity we argue as follows.

Let $F(t)=\sum_{m, q \in \mathbb{Z}_{+}^{N}} F_{m q}(t) a^{\dagger m} a^{q}$. Using (1.1), (1.3), 1.6),

$$
\begin{aligned}
& \frac{d}{d t}\left\langle\Phi_{\alpha} \mid F \Phi_{\alpha}\right\rangle \\
& =\frac{i}{\hbar}\left\langle\Phi_{\alpha} \mid(H F-F H) \Phi_{\alpha}\right\rangle \\
& =\frac{i}{\hbar} \sum_{\ell, s, m, q} H_{\ell s} F_{m q}(t)\left\{\left\langle\Phi_{\alpha} \mid a^{\dagger \ell} a^{s} a^{\dagger m} a^{q} \Phi_{\alpha}\right\rangle-\left\langle\Phi_{\alpha} \mid a^{\dagger m} a^{q} a^{\dagger \ell} a^{s} \Phi_{\alpha}\right\rangle\right\} \\
& =\frac{i}{\hbar} \sum_{\ell, s, m, q} H_{\ell s} F_{m q}\left\{\alpha^{* \ell} \alpha^{q}\left\langle a^{\dagger s} \Phi_{\alpha} \mid a^{\dagger m} \Phi_{\alpha}\right\rangle-\alpha^{* m} \alpha^{s}\left\langle a^{\dagger q} \Phi_{\alpha} \mid a^{\dagger \ell} \Phi_{\alpha}\right\rangle\right\} \\
& =\frac{i}{\hbar} \sum_{\ell, s, m, q} H_{\ell s} F_{m q}\left\{\alpha^{* \ell} \alpha^{q}\left(\hbar \frac{\partial}{\partial \alpha^{*}}\right)^{s}\left(\hbar \frac{\partial}{\partial \alpha}\right)^{m}-\alpha^{* m} \alpha^{s}\left(\hbar \frac{\partial}{\partial \alpha^{*}}\right)^{q}\left(\hbar \frac{\partial}{\partial \alpha}\right)^{\ell}\right\} \exp \frac{|\alpha|^{2}}{\hbar} \\
& =\frac{i}{\hbar} \sum_{\ell, s, m, q} H_{\ell s} F_{m q}\left\{\alpha^{* \ell}\left(\hbar \frac{\partial}{\partial \alpha^{*}}\right)^{s} \alpha^{* m} \alpha^{q}-\alpha^{s}\left(\hbar \frac{\partial}{\partial \alpha}\right)^{\ell} \alpha^{* m} \alpha^{q}\right\} \exp \frac{|\alpha|^{2}}{\hbar} \\
& =\frac{i}{\hbar} \sum_{\ell, s} H_{\ell s}\left\{\alpha^{* \ell}\left(\hbar \frac{\partial}{\partial \alpha^{*}}\right)^{s}-\alpha^{s}\left(\hbar \frac{\partial}{\partial \alpha}\right)^{\ell}\right\} \exp \frac{|\alpha|^{2}}{\hbar}\langle\alpha|F(t)| \alpha\rangle \text {. }
\end{aligned}
$$

In a slightly more general setting, let

$$
H=\text { op }^{\text {Wick }} \mathcal{H}\left(z^{*}, z\right)
$$

For $H$ as in (1.1) this means

$$
\mathcal{H}\left(z^{*}, z\right)=\sum_{\ell, s} H_{\ell s} z^{* \ell} z^{s}
$$

Then from (1.5), (1.7) we get a closed equation for the evolution of $\langle\alpha|F| \alpha\rangle$ :

$$
\begin{aligned}
& \frac{d}{d t}\langle\alpha|F| \alpha\rangle= \\
& \quad=\frac{i}{\hbar} \text { op }\left[\left(\mathcal{H}\left(\alpha^{*}, \frac{1}{2} \hbar \xi+\alpha\right)-\mathcal{H}\left(\frac{1}{2} \hbar \xi^{*}+\alpha^{*}, \alpha\right)\right)\right]\langle\alpha|F| \alpha\rangle
\end{aligned}
$$


Here, as in the theory of pseudodifferential operators, for any appropriate symbol $b(\alpha, \xi)$

$$
\mathrm{op}[b(\alpha, \xi)] f=(2 \pi)^{-2 N} \int_{\mathbb{C}^{N}} \int_{\mathbb{C}^{N}} b(\alpha, \xi) e^{i \operatorname{Re} \xi \cdot(\alpha-\gamma)^{*}} f(\gamma) d^{2 N} \gamma d^{2 N} \xi
$$

For the purpose of the present paper it will be sufficient to treat the case when $\mathcal{H}$ is a polynomial in $z^{*}, z$. We will address the mathematical problems related to a more general $\mathcal{H}$ in future publications.

In case $\mathcal{H}\left(z^{*}, z\right)$ is a polynomial, the equation (1.9) has the following explicit form:

$$
\begin{aligned}
& \frac{d}{d t}\langle\alpha|F| \alpha\rangle= \\
& \quad=\frac{i}{\hbar} \sum_{r \in \mathbb{Z}_{+}^{N}} \frac{1}{r !}\left(\left(\frac{\partial}{\partial \alpha}\right)^{r} \mathcal{H}\left(\alpha^{*}, \alpha\right)\left(\hbar \frac{\partial}{\partial \alpha^{*}}\right)^{r}-\left(\frac{\partial}{\partial \alpha^{*}}\right)^{r} \mathcal{H}\left(\alpha^{*}, \alpha\right)\left(\hbar \frac{\partial}{\partial \alpha}\right)^{r}\right)\langle\alpha|F| \alpha\rangle
\end{aligned}
$$

Here, for $r=\left(r_{1}, \ldots, r_{N}\right) \in \mathbb{Z}_{+}^{N}, r !=r_{1} ! \cdots r_{N} !$

2.

The goal of the paper is to present an asymptotic solution to $(1.9),(1.10)$ as $\hbar \rightarrow 0$. We first treat the case of the initial condition of the following nature:

$$
\langle\alpha|F(0)| \alpha\rangle=\alpha^{* m} \alpha^{q} \exp \left(-\frac{\left|\alpha-\alpha_{0}\right|^{2}}{\hbar}\right), \quad \alpha_{0} \in \mathbb{C}^{N} .
$$

This corresponds, i.e., to the choice

$$
F(0)=a^{\dagger m}\left|\alpha_{0}\right\rangle\left\langle\alpha_{0}\right| a^{q}
$$

Indeed, for $\alpha \in \mathbb{C}^{N}$,

$$
\begin{aligned}
\left\langle\alpha\left|a^{\dagger m}\right| \alpha_{0}\right\rangle\left\langle\alpha_{0}\left|a^{q}\right| \alpha\right\rangle & =\alpha^{* m} \alpha^{q}\left|\left\langle\alpha_{0}, \alpha\right\rangle\right|^{2} \\
& =\alpha^{* m} \alpha^{q}\left|\exp \left(\frac{\alpha_{0}^{*} \alpha}{\hbar}-\frac{\left|\alpha_{0}\right|^{2}}{2 \hbar}-\frac{|\alpha|^{2}}{2 \hbar}\right)\right|^{2} \\
& =\alpha^{* m} \alpha^{q} \exp \left(-\frac{\left|\alpha-\alpha_{0}\right|^{2}}{\hbar}\right) .
\end{aligned}
$$

To solve asymptotically the problem (1.9), (1.10) with the initial condition as in (2.1) we propose the following procedure. Let $f\left(\alpha^{*}, \alpha, t\right)=\langle\alpha|F(t)| \alpha\rangle$.

Assume formally

$$
f\left(\alpha^{*}, \alpha, t\right)=e^{\frac{S\left(\alpha^{*}, \alpha, t\right)}{\hbar}} \sum_{j=0}^{\infty} b_{j}\left(\alpha^{*}, \alpha, t\right) \hbar^{j},
$$

where the phase $S\left(\alpha^{*}, \alpha, t\right)$ and the coefficients $b_{j}, j=0,1,2, \ldots$ are to be determined.

The initial conditions are

$$
\begin{aligned}
S\left(\alpha^{*}, \alpha, 0\right) & =-\left|\alpha-\alpha_{0}\right|^{2} \\
b_{0}\left(\alpha^{*}, \alpha, 0\right) & =\alpha^{* m} \alpha^{q} ; \\
b_{j}\left(\alpha^{*}, \alpha, 0\right) & =0 \text { for } j \geq 1 .
\end{aligned}
$$

With these choices (2.1) will follow immediately. We substitute (2.3) into (1.10) and obtain the Hamilton-Jacobi equation for $S$ and a system of transport equations for $b_{j}, j \geq 0$.

For the phase $S$ we have from Taylor's formula:

$$
\dot{S}=i\left\{\mathcal{H}\left(\alpha^{*}, \alpha+\frac{\partial S}{\partial \alpha^{*}}\right)-\mathcal{H}\left(\alpha^{*}+\frac{\partial S}{\partial \alpha}, \alpha\right)\right\}
$$


It is a rather remarkable fact that the Hamilton-Jacobi equation (2.7) is real (see (1.2)) and therefore the classical Hamilton-Jacobi theory applies. The expansion (2.3) describes Laplace asymptotics unlike WKB asymptotics valid for the Schrödinger equation (see, e.g., 8]) describing the evolution of a wave function.

We introduce the momenta $p, p^{*}$ and the effective Hamiltonian associated with (2.7) as follows:

$$
W\left(\alpha^{*}, \alpha, p^{*}, p\right)=-i\left\{\mathcal{H}\left(\alpha^{*}, \alpha+p^{*}\right)-\mathcal{H}\left(\alpha^{*}+p, \alpha\right)\right\} .
$$

The Hamiltonian dynamics in these variables is

$$
\left\{\begin{array}{l}
\dot{\alpha}_{k}=i \frac{\partial \mathcal{H}}{\partial \alpha_{k}^{*}}\left(\alpha^{*}+p, \alpha\right) \\
\dot{p}_{k}=i\left\{\frac{\partial \mathcal{H}}{\partial \alpha_{k}}\left(\alpha^{*}, \alpha+p^{*}\right)-\frac{\partial \mathcal{H}}{\partial \alpha_{k}}\left(\alpha^{*}+p, \alpha\right)\right\} \\
\dot{\alpha}_{k}^{*}=-i \frac{\partial \mathcal{H}}{\partial \alpha_{k}}\left(\alpha^{*}, \alpha+p^{*}\right) \\
\dot{p}_{k}^{*}=i\left\{\frac{\partial \mathcal{H}}{\partial \alpha_{k}^{*}}\left(\alpha^{*}, \alpha+p^{*}\right)-\frac{\partial \mathcal{H}}{\partial \alpha_{k}^{*}}\left(\alpha^{*}+p, \alpha\right)\right\}
\end{array}\right.
$$

We now fix $\alpha_{0} \in \mathbb{C}^{N}$ and solve the equation (2.7). Let

$$
\begin{aligned}
\mathrm{E}\left(\alpha^{*}, \alpha, p^{*}, p\right)=i\{ & \left(p \frac{\partial \mathcal{H}}{\partial \alpha^{*}}\left(\alpha^{*}+p, \alpha\right)-p^{*} \frac{\partial \mathcal{H}}{\partial \alpha}\left(\alpha^{*}, \alpha+p^{*}\right)\right) \\
& \left.-\mathcal{H}\left(\alpha^{*}+p, \alpha\right)+\mathcal{H}\left(\alpha^{*}, \alpha+p^{*}\right)\right\}
\end{aligned}
$$

Denote by $g^{t}$ the Hamiltonian dynamics corresponding to

$$
\dot{\alpha}_{k}=i \frac{\partial \mathcal{H}}{\partial \alpha_{k}^{*}}\left(\alpha^{*}, \alpha\right), \quad k=1, \ldots, N .
$$

Under the condition $\left|\mathcal{H}\left(\alpha^{*}, \alpha\right)\right| \geq c_{1}|\alpha|^{M}-c_{2}$, where $M>0, c_{1}>0, c_{2}>0$ this dynamics is defined for all $\alpha(0) \in \mathbb{R}^{N}$, $t \in \mathbb{R}$

$$
\alpha=g^{t}(\alpha(0))
$$

and is a 1-parametric group of (real)-analytic diffeomorphisms $\mathbb{C}^{N} \stackrel{\sim}{\rightarrow} \mathbb{C}^{N} \cdot 14$

Let $\alpha$ belong to a small enough neighborhood of $g^{t} \alpha_{0}$. We solve (2.9) with initial data

$$
\begin{cases}\alpha_{k}(0), & k=1, \ldots, N \\ p_{k}(0)=-\left(\alpha_{k}^{*}(0)-\alpha_{0 k}^{*}\right), & k=1, \ldots, N\end{cases}
$$

Here $\alpha(0)$ is chosen in a small neighborhood of $\alpha_{0}$ in $\mathbb{C}^{N}$.

Linearizing (2.9) along a trajectory with $p=0$, we obtain

$$
\left\{\begin{array}{l}
\dot{A}_{k}=i\left\{\frac{\partial^{2} \mathcal{H}}{\partial \alpha_{k}^{*} \partial \alpha_{r}}\left(\alpha^{*}, \alpha\right) A_{r}+\frac{\partial^{2} \mathcal{H}}{\partial \alpha_{k}^{*} \partial \alpha_{r}^{*}}\left(\alpha^{*}, \alpha\right)\left(A_{r}^{*}+B_{r}\right)\right\} \\
\dot{B}_{k}=i\left\{\frac{\partial^{2} \mathcal{H}}{\partial \alpha_{k} \partial \alpha_{r}}\left(\alpha^{*}, \alpha\right) B_{r}^{*}-\frac{\partial^{2} \mathcal{H}}{\partial \alpha_{k} \partial \alpha_{r}^{*}}\left(\alpha^{*}, \alpha\right) B_{r}\right\}
\end{array}\right.
$$

where $A_{k}=\delta \alpha_{k}, B_{k}=\delta p_{k}, k=1, \ldots, N$.

From (2.10) the initial condition for (2.11) satisfies

$$
B_{k}(0)=-A_{k}^{*}(0), \quad k=1, \ldots, N .
$$

We want to show that the map that assigns $\alpha(t)$ to each $\alpha(0)$ in a small neighborhood of $\alpha_{0}$ where $(\alpha(t), p(t))$ solves (2.9) with initial condition (2.10) is a local diffeomorphism (of class $C^{\omega}$ ). Once this is established, $S$ can be defined in a small neighborhood of $g^{t} \alpha_{0}$ as follows:

$$
S\left(\alpha^{*}(t), \alpha(t), t\right)=-\left|\alpha(0)-\alpha_{0}\right|^{2}+\int_{0}^{t} \mathrm{E}\left(\alpha^{*}(\tau), \alpha(\tau), p^{*}(\tau), p(\tau)\right) d \tau
$$


Also, $p^{*}(t)=\frac{\partial S}{\partial \alpha^{*}}\left(\alpha^{*}(t), \alpha(t), t\right), p(t)=\frac{\partial S}{\partial \alpha}\left(\alpha^{*}(t), \alpha(t), t\right)$. To verify the statement above we notice that (2.11) yields

$$
\begin{aligned}
\frac{d}{d t}\left(A_{k} B_{k}+A_{k}^{*} B_{k}^{*}\right)= & i\left\{\frac{\partial^{2} \mathcal{H}}{\partial \alpha_{k}^{*} \partial \alpha_{r}} A_{r} B_{k}+\frac{\partial^{2} \mathcal{H}}{\partial \alpha_{k}^{*} \partial \alpha_{r}^{*}}\left(A_{r}^{*}+B_{r}\right) B_{k}+\frac{\partial^{2} \mathcal{H}}{\partial \alpha_{k} \partial \alpha_{r}} A_{k} B_{r}^{*}-\frac{\partial^{2} \mathcal{H}}{\partial \alpha_{k} \partial \alpha_{r}^{*}} A_{k} B_{r}\right\} \\
& -i\left\{\frac{\partial^{2} \mathcal{H}}{\partial \alpha_{r}^{*} \partial \alpha_{k}} A_{r}^{*} B_{k}^{*}+\frac{\partial^{2} \mathcal{H}}{\partial \alpha_{r} \partial \alpha_{k}}\left(A_{r}+B_{r}^{*}\right) B_{k}^{*}+\frac{\partial^{2} \mathcal{H}}{\partial \alpha_{r}^{*} \partial \alpha_{k}^{*}} A_{k}^{*} B_{r}-\frac{\partial^{2} \mathcal{H}}{\partial \alpha_{r} \partial \alpha_{k}^{*}} A_{k}^{*} B_{r}^{*}\right\} \\
= & i \frac{\partial^{2} \mathcal{H}}{\partial \alpha_{k}^{*} \partial \alpha_{r}^{*}} B_{r} B_{k}-i \frac{\partial^{2} \mathcal{H}}{\partial \alpha_{r} \partial \alpha_{k}} B_{r}^{*} B_{k}^{*} \\
= & -\frac{d}{d t} B_{k} B_{k}^{*}
\end{aligned}
$$

Integrating and using (2.12) we get

$$
A_{k}(t) B_{k}(t)+A_{k}^{*}(t) B_{k}^{*}(t)+B_{k}(t) B_{k}^{*}(t)=-2|B(0)|^{2}+|B(0)|^{2}=-|B(0)|^{2} .
$$

this implies $A_{k}(t) B_{k}(t)+A_{k}^{*}(t) B_{k}^{*}(t) \leq-|B(t)|^{2}$. By using the second equation $(2.11)$ and $(2.12)$ we get $C|A(0)| \geq$ $|B(t)| \geq C^{-1}|A(0)|$ with some $C>0$ uniform over any finite time interval $[0, T]$. Therefore, $|A(t)| \geq C^{-1}|A(0)|$ and one would never encounter a caustic moving along the trajectory (2.9) with $p=0$ (i.e., along $g^{t} \alpha_{0}$ ). This is enough to conclude. The solution (2.13) to (2.7), (2.4) is unique in the obvious sense.

Clearly $S\left(\left(g^{t} \alpha_{0}\right)^{*}, g^{t} \alpha_{0}, t\right)=0$ and

$$
\frac{\partial S}{\partial \alpha^{*}}\left(\left(g^{t} \alpha_{0}\right)^{*}, g^{t} \alpha_{0}, t\right)=0, \quad \frac{\partial S}{\partial \alpha}\left(\left(g^{t} \alpha_{0}\right)^{*}, g^{t} \alpha_{0}, t\right)=0
$$

Also, the function $S\left(\alpha^{*}, \alpha, t\right)$ satisfies the inequality

$$
S\left(\alpha^{*}, \alpha, t\right) \leq-C\left|\alpha-g^{t} \alpha_{0}\right|^{2}
$$

in some possibly smaller neighborhood of $g^{t} \alpha_{0}$. This follows from the above argument since $A_{k}(t) B_{k}(t)+A_{k}^{*}(t) B_{k}^{*}(t)$ is the second differential of $S(t)$ at the critical point $\left(\left(g^{t} \alpha_{0}\right)^{*}, g^{t} \alpha_{0}\right)$.

3.

We now turn to the system of transport equations for the coefficients $b_{j}\left(\alpha^{*}, \alpha, t\right), j \geq 0$. We have, substituting (2.3) into (1.10) and collecting terms of order $\hbar^{0}$ :

$$
\begin{gathered}
\dot{b}_{0}=i \sum_{r \in \mathbb{Z}_{+}^{N}} \sum_{\ell=1}^{N} \frac{r_{\ell}}{r !}\left\{\left(\frac{\partial}{\partial \alpha}\right)^{r} \mathcal{H}\left(\alpha^{*}, \alpha\right)\left[\left(\frac{\partial S}{\partial \alpha^{*}}\right)^{r-1} \frac{\partial}{\partial \alpha_{\ell}^{*}} b_{0}+\frac{1}{2} \frac{\partial}{\partial \alpha_{\ell}^{*}}\left(\left(\frac{\partial S}{\partial \alpha^{*}}\right)^{r-1_{\ell}}\right) b_{0}\right]\right. \\
\left.-\left(\frac{\partial}{\partial \alpha^{*}}\right)^{r} \mathcal{H}\left(\alpha^{*}, \alpha\right)\left[\left(\frac{\partial S}{\partial \alpha}\right)^{r-1} \frac{\partial}{\partial \alpha_{\ell}} b_{0}+\frac{1}{2} \frac{\partial}{\partial \alpha_{\ell}}\left(\left(\frac{\partial S}{\partial \alpha}\right)^{r-1}\right) b_{0}\right]\right\} .
\end{gathered}
$$

In other words,

$$
\begin{aligned}
& \dot{b}_{0}=i \sum_{\ell=1}^{N}\left\{\frac{\partial}{\partial \alpha_{\ell}} \mathcal{H}\left(\alpha^{*}, \alpha+\frac{\partial S}{\partial \alpha^{*}}\right) \frac{\partial}{\partial \alpha_{\ell}^{*}}-\frac{\partial}{\partial \alpha_{\ell}^{*}} \mathcal{H}\left(\alpha^{*}+\frac{\partial S}{\partial \alpha}, \alpha\right) \frac{\partial}{\partial \alpha_{\ell}}\right\} b_{0} \\
&+\frac{i}{2} \sum_{\ell=1}^{N} \sum_{m=1}^{N}\left\{\frac{\partial^{2}}{\partial \alpha_{\ell} \partial \alpha_{m}} \mathcal{H}\left(\alpha^{*}, \alpha+\frac{\partial S}{\partial \alpha^{*}}\right) \frac{\partial^{2} S}{\partial \alpha_{\ell}^{*} \partial \alpha_{m}^{*}}\right. \\
&\left.\quad-\frac{\partial^{2}}{\partial \alpha_{\ell}^{*} \partial \alpha_{m}^{*}} \mathcal{H}\left(\alpha^{*}+\frac{\partial S}{\partial \alpha}, \alpha\right) \frac{\partial^{2} S}{\partial \alpha_{\ell} \partial \alpha_{m}}\right\} b_{0} \\
& \equiv L_{0} b_{0}
\end{aligned}
$$

In the right side of (3.1) $1_{\ell}, \ell=1, \ldots, N$ is the vector with components $\delta_{\ell s}, s=1, \ldots, N$. 
For an arbitrary $j \geq 0$

$$
\dot{b}_{j}=L_{0} b_{j}+\sum_{k=1}^{j} L_{k} b_{j-k} .
$$

The differential operators $L_{k}$ in the right hand side of (3.3) are as follows:

$$
\begin{gathered}
L_{k}=\frac{i}{(k+1) !} \frac{\partial^{k+1}}{\partial \hbar^{k+1}} \sum_{r \in \mathbb{Z}_{+}^{N}} \frac{1}{r !}\left\{\left(\frac{\partial}{\partial \alpha}\right)^{r} \mathcal{H}\left(\alpha^{*}, \alpha\right)\left(\hbar \frac{\partial}{\partial \alpha^{*}}+\frac{\partial S}{\partial \alpha^{*}}\right)^{r}\right. \\
\left.-\left(\frac{\partial}{\partial \alpha^{*}}\right)^{r} \mathcal{H}\left(\alpha^{*}, \alpha\right)\left(\hbar \frac{\partial}{\partial \alpha}+\frac{\partial S}{\partial \alpha}\right)^{r}\right\}\left.\right|_{\hbar=0}
\end{gathered}
$$

To solve the system (3.1)-(3.4) with initial conditions (2.5), (2.6) we use (2.9) to obtain with fixed $\alpha^{*}(0), \alpha(0)$ in a small neighborhood of $\alpha_{0} \in \mathbb{C}^{N}$

$$
\frac{d}{d t} b_{0}\left(\alpha^{*}(t), \alpha^{*}(t), t\right)+c_{0}\left(\alpha^{*}(t), \alpha(t), t\right) b_{0}=0
$$

where $c_{0}$ is the coefficient in the second term of (3.2). Having solved (3.5) with initial condition (2.5), we obtain a first order linear inhomogeneous ODE for $b_{1}$, etc.. According to (3.3) to solve the equation for $b_{j}$ we are recursively faced with the problem of solving the same ODE with a different known right hand side. The solution to this problem therefore exists and is unique for $\left(\alpha^{*}, \alpha\right)$ being sufficiently close to $\left(\left(g^{t} \alpha_{0}\right)^{*}, g^{t} \alpha_{0}\right)$.

To solve the problem (1.9), (1.10) with the initial condition

$$
\langle\alpha|F(0)| \alpha\rangle=\alpha^{* m} \alpha^{q}
$$

we use the completeness relation

$$
\frac{1}{(\pi \hbar)^{N}} \int\left|\alpha_{0}\right\rangle\left\langle\alpha_{0}\right| d^{2 N} \alpha_{0}=i d
$$

which leads to the expansion

$$
\langle\alpha|F(t)| \alpha\rangle=\frac{1}{(\pi \hbar)^{N}} \int d^{2 N} \alpha_{0} e^{\frac{S\left(\alpha^{*}, \alpha, t\right)}{\hbar}} \sum_{j=0}^{\infty} b_{j}\left(\alpha^{*}, \alpha, t\right) \hbar^{j}
$$

Here in the right hand side the phase $S$ and the coefficients $b_{j}, j \geq 0$ depend on a parameter $\alpha_{0} \in \mathbb{C}^{N}$.

\section{Example (see [12], [8] for the exact solution)}

Let $N=1, \mathcal{H}\left(\alpha^{*}, \alpha\right)=\omega \alpha^{*} \alpha+\mu \alpha^{* 2} \alpha^{2}$. The equation (2.7) takes the form

$$
\dot{S}=i\left(\omega+2 \mu|\alpha|^{2}\right)\left(\alpha^{*} \frac{\partial S}{\partial \alpha^{*}}-\alpha \frac{\partial S}{\partial \alpha}\right)+i \mu\left(\alpha^{* 2}\left(\frac{\partial S}{\partial \alpha^{*}}\right)^{2}-\alpha^{2}\left(\frac{\partial S}{\partial \alpha}\right)^{2}\right)
$$

The first transport equation is

$$
\begin{aligned}
\dot{b}_{0}= & i\left(\omega+2 \mu|\alpha|^{2}\right)\left(\alpha^{*} \frac{\partial}{\partial \alpha^{*}}-\alpha \frac{\partial}{\partial \alpha}\right) b_{0}+2 i \mu\left(\alpha^{* 2} \frac{\partial S}{\partial \alpha^{*}} \frac{\partial}{\partial \alpha^{*}}-\alpha^{2} \frac{\partial S}{\partial \alpha} \frac{\partial}{\partial \alpha}\right) b_{0} \\
& +i \mu\left(\alpha^{* 2} \frac{\partial^{2} S}{\partial \alpha^{* 2}}-\alpha^{2} \frac{\partial^{2} S}{\partial \alpha^{2}}\right) b_{0} \equiv L_{0} b_{0}
\end{aligned}
$$

A simple computation yields:

$$
\begin{gathered}
S\left(\alpha^{*}, \alpha, t\right)=-\left|\alpha(0)-\alpha_{0}\right|^{2}-i \mu t\left(\alpha^{*}(0)^{2} \alpha_{0}^{2}-\alpha(0)^{2} \alpha_{0}^{* 2}\right) \\
+|\alpha(0)|^{2}\left(1-e^{-2 i \mu t\left(\alpha^{*}(0) \alpha_{0}-\alpha(0) \alpha_{0}^{*}\right)}\right) \\
b_{0}\left(\alpha^{*}, \alpha, t\right)=\alpha(0)^{* m} \alpha(0)^{q} \frac{1}{\sqrt{1+4 \mu^{2} t^{2}\left|\alpha_{0}\right|^{2}|\alpha(0)|^{2}}} \\
\exp \left\{\frac{i\left(\alpha(0)^{*} \alpha_{0}-\alpha(0) \alpha_{0}^{*}\right)}{2|\alpha(0)|\left|\alpha_{0}\right|} \arctan \left(2 \mu t\left|\alpha_{0}\right||\alpha(0)|\right)\right\},
\end{gathered}
$$


where

$$
\alpha=\alpha(0) \exp \left\{i \omega t+2 \mu t \alpha_{0}^{*} \alpha(0)\right\}
$$

Notice that for any $t$ the map (4.5) $\alpha(0) \rightarrow \alpha$ is invertible in a sufficiently small neighborhood of $\alpha_{0}$.

\section{Conclusion}

In conclusion, we developed a theory for Bose systems with many degrees of freedom which describes not an oscillating asymptotic behavior of a quasi-classical wave function, but a Laplace asymptotics for quantum average quantities, which are localized in phase space. This approach has many advantages in comparison with the standard one, as it does not require (i) an intermideate step of computing a complicated behaviour of a quasi-classical wave function which usually presents difficulties associated with caustics, and (ii) an additinal integration for computing the average values. Our approach can be used for solving many dynamical problems related to Bose systems with many degrees of freedom.

\section{Acknowledgements}

This work was supported by the Department of Energy under the contract W-7405-ENG-36 and DOE Office of Basic Energy Sciences. The work of MV was supported in part by the National Science Foundation Grant DMS-9876947. The work of GPB was partly supported by the National Security Agency (NSA) and by the Advanced Research and Development Activity (ARDA). MV is grateful to the Los Alamos National Laboratory for the kind hospitality during his visit in July 2002.

* vishik@math.utexas.edu

† gpb@lanl.gov

[1] R. Glauber, Phys. Rev. 131, 2766 (1963).

[2] M. Gutzwiller, Chaos in Classical and Quantum Mechanics (Springer, 1990).

[3] L. Reichl, The Transition to Chaos (Springer-Verlag, 1992).

[4] F. Berezin and M. Shubin, The Schrödinger Equation (Kluwer, 1991).

[5] G. Agarval and E. Wolf, Phys. Rev. D 2, 2161; 2187; 2206 (1970).

[6] P. Caruthers and M. Nieto, Rev. Mod. Phys. 40, 411 (1968).

[7] Y. Sinitsyn and V. Tsukernik, Phys. Lett. A 90, 399 (1982).

[8] G. Berman, E. Bulgakov, and D. Holm, Crossover-Time in Quantum Boson and Spin Systems (Springer-Verlag, 1994).

[9] V. Maslov, Théory des Perturbations et Méthodes Asymptotiques (Dunod, Paris, 1972).

[10] V. Maslov, Operator Methods (Mir, Moscow, 1982 (Russian)).

[11] L. Hörmander, The Analysis of Linear Partial Differential Operators, vol. 1-4 (Springer, Berlin, 1983-1985).

[12] G. Berman, A. Iomin, and G. Zaslavsky, Physica D 4, 113 (1981).

[13] M. Shubin, Pseudodifferential Operators and Spectral Theory (Springer, Berlin, 1987).

[14] To ensure the well-posedness of the evolution (1.3) one needs some additional assumptions on $\mathcal{H}$. The following condition suffices (see [11], [13]). There exist $R>0, \rho>0$ and a set of constants $C_{s \ell}$, where $s, \ell \in \mathbb{Z}_{+}^{N}$, so that

$$
\left|\frac{\partial^{|s+\ell|}}{\partial \alpha^{* s} \partial \alpha^{\ell}} \mathcal{H}\left(\alpha^{*}, \alpha\right)\right| \leq C_{s, \ell}|\alpha|^{-\rho|s+\ell|}\left|\mathcal{H}\left(\alpha^{*}, \alpha\right)\right|, \quad|\alpha| \geq R .
$$

